\title{
Resumos dos Temas-Livres apresentados ao IV Congresso Brasileiro de Ergometria e Reabilitação Cardiovascular - 30/10 a 1/11/97 - Ribeirão Preto-SP
}

\section{CARACTERÍSTICAS DA POPULAÇÃO ATENDIDA NO PROGRAMA DE CONDICIONAMENTO FÍSICO APLICADO À PREVENÇÃO CARDIOLÓGICA PRIMÁRIA E SECUNDÁRIA DA ESCOLA DE EDUCAÇÃO FÍSICA E ESPORTE DA USP E DO INSTITUTO DO CORAÇÃO HC- FMUSP.}

\author{
Souza MO, Coelho MA, Rondon MUPB, Barreto ACP, Negrão CE, Forjaz CLM.
}

Escola de Educação Física e Esporte da USP e Serviço de Condicionamento Físico do InCor HC-FMUSP, São Paulo, SP.

A prescrição individualizada de exercícios, levando em conta as características da população atendida, tem sido amplamente recomendada. No entanto, a caracterização das populações atendidas em programas de condicionamento físico para a prevenção cardiológica (PCF) tem sido pouco descrita. Assim, este estudo teve por objetivo caracterizar a população participante do nosso PCF. Para tanto, 214 alunos participantes do PCF foram classificados quanto ao: sexo, idade (jovem: 20-40; meia idade: 41-60 e idoso: >60), índice de massa corporal (normal: IMC $<27,0$ e sobrepeso IMC $\geq 27$ ) e diagnóstico (preventivos ou cardiopatas). Considerou-se também o período em que os indivíduos frequentavam o programa (manhã e noite). Na população total predominaram os homens (65\%) de meia idade $(54 \%$ ) e preventivos (58\%). Quando se comparou os grupos de cardíacos e preventivos observou-se diferença em relação ao sexo e idade, ou seja, nos cardíacos houve predominância do sexo masculino (74\%) e dos idosos (59\%), enquanto nos preventivos não houve predominância de sexo (homens $=58 \%$ e mulheres $=42 \%$ ) e houve predominância de meia-idade (63\%). Quanto ao diagnóstico, dentre os cardíacos a maioria apresentou insuficiência coronariana $(78 \%)$ e nos preventivos a maioria apresentou hipertensão arterial sistêmica (HAS-47\%). O IMC normal e sobrepeso não predominaram em nenhum dos grupos analisados. É interessante ressaltar que dentre os períodos da manhã e da noite houve diferença no diagnóstico e na faixa etária atendida, de modo que, no período da noite predominaram os indivíduos preventivos (73\%) e de meia idade (61\%) e no período da manhã predominaram os indivíduos idosos $952 \%$ ) e não houve diferença entre os diagnósticos (55\% Cardiopatas e $45 \%$ Preventivos). Em conclusão, o nosso PFC atende predominantemente indivíduos do sexo masculino em prevenção primária, sendo a HAS o principal fator de risco encontrado. Além disso, os indivíduos cardiopatas e idosos realizam suas atividades físicas preferencialmente no período da manhã, enquanto os preventivos de meia idade no período da noite. Esses resultados, com certeza, poderão melhor nortear a prescrição individualizada de exercícios no nosso PCF.

\section{INFLUÊNCIA DO GÊNERO NO TIPO DE STRESS UTILIZADO PARA A CINTOLOGRAFIA DE PERFUSÃO MIOCÁRDIA}

AUTOR: Cardoso Fo, M.S

CO-AUTORES: Souza Fo, J.; Fahel, V.G.; Lobão, L.J.; Costa, E.; Lopes, A.A.

Serviços de Cardiologia, Medicina Nuclear e Setor de Epidemiologia Clínica e Bioestatística do Hospital São Rafael - Salvador - BA

Objetivos: Ultimamente têm aparecido diversas publicações sobre diferenças entre homens e mulheres no que se refere a indicações de procedimentos diagnósticos e terapêuticos na área de cardiologia. Estas questões, no entanto, têm sido pouco exploradas aqui no Brasil. Por essa razão, este estudo foi desenvolvido com o propósito básico de investigar prováveis diferenças entre homens e mulheres no tipo de stress (farmacológico com dipiridamol vs teste ergométrico) utilizado para a realização de cintilografia de perfusão miocárdica (CPM) mesmo após o ajuste para a variável idade.

Métodos: Foram levantadas informações quanto ao sexo, idade ( $<=50 ;>50$ anos) e tipo de stress de todos os 1.735 pacientes submetidos a C.P.M. no Serviço de Medicina Nuclear do Hospital de São Rafael, entre março de 1992 a dezembro de 1996. utilizou-se o teste t de Student e o qui-quadrado para os testes estatísticos de diferenças de médias e proporções, respectivamente. As estimativas das razões de freqüência (RF) da utilização de dipiridamol entre homens e mulheres foram descritas atavés das estimativas pontuais e intervalos de confiança de 95\% (IC95\%). Utilizouse o SPSS v.6 para as análises estatísticas.

Resultados: Do total de pacientes, $793(45,7 \%)$ eram do sexo feminino. As mulheres foram significativamente $(\mathrm{p}=0,004)$ mais velhas $(57,3 \pm 10,7$ anos $)$ do que os homens $(55,8 \pm 11,5)$, sendo que $71,4 \%$ das mulheres e $65,8 \%$ dos homens tinham idade $>50$ anos. O teste com dipiridamol foi realizado em 61,3 das mulheres e $41,2 \%$ dos homens $(R F=1,49 ;$ IC $95 \%=1,4-1,6)$, sendo portanto o uso do stress farmacológico aproximadamente $49 \%$ mais freqüente nas mulheres do que nos homens. As comparações entre homens e mulheres ao tipo de stress foram similares entre os grupos etários $<=50$ anos ( $R F=1,49$; IC $95 \%=1,16-1)$, e $>50$ anos (RF $=1,43 \% ;$ IC $95 \%=1,3-1,57$ )

Conclusões: O stress farmacológico com dipiridamol foi mais freqüentemente utilizado como forma de stress para CPM nas mulheres do que nos homens, independente da faixa etária. As razões pela preferência por este tipo de stress nas mulheres não puderam ser esclarecidos por este estudo, permanecendo como uma importante questão para futuras investigações.

\section{QUALIDADE DE VIDA E NÍVEL DE STRESS DE INGRESSANTES E PARTICIPANTES REGULARES DO PROGRAMA DE CONDICIONAMENTO FÍSICO APLICADO À CARDIOLOGIA PRIMÁRIA E SECUNDÁRIA DA ESCOLA DE EDUCAÇÃO FÍSICA E ESPORTE DA USP E DO INSTITUTO DO CORAÇÃO, HC-FMUSP.}

Mara R.F. Caruso, Bellkiss W. Romano, Carlos E. Negrão, Antonio C.P. Barreto, Cláudia L.M. Forjaz.

Escola de Educação Física e Esporte USP e Serviço de Condicionamento Físico e Serviço de Psicologia do InCor-HCFMUSP.

A importância do condicionamento físico na qualidade de vida e no controle do stress tem sido muito divulgada. No entanto, poucos estudos científicos abordaram de forma direta e específica essa problemática. Assim, este estudo teve por objetivo comparar a qualidade de vida, o nível de stress e a ansiedade de indivíduos sedentários e participantes de um programa de condicionamento físico (PCF). Foram estudados 39 indivíduos preventivos entre 40 e 60 anos, sendo 19 participantes regulares (PR) do PCF e 20 sedentários ingressantes (PI) nesse programa. A avaliação consistiu na aplicação individual de: 1) inventário de Qualidade de Vida (esfera social / afetiva / profissional / saúde); 2) inventário de Sintomas de Stress (fases de alerta, resistência e exaustão); 3) teste de Características da Personalidade Geradoras de Stress (forma de pensar e agir / assertividade / afetividade); 4) Teste Idate para medir ansiedade (estado / traço) e; 5) Teste de Mackover da figura humana para identificar personalidade patológica. Nenhum dos indivíduos apresentou personalidade patológica. Não houve diferença significante na qualidade de vida dos dois grupos, mas a qualidade de vida profissional tendeu a ser maior no grupo PR que $\mathrm{PI}(5,7 \pm 1,0$ vs $4,5 \pm 1,7, \mathrm{p}=0,08)$. Além disso, o grupo $\mathrm{PR}$ apresentou uma tendência a ter menos sintomas de stress $(4,0 \pm 3,5$ vs. $7,0 \pm 5,9, P=0,06)$ que o grupo $\mathrm{PI}$ e essa diferença foi significante para os sintomas de exaustão ( $P R=0,7 \pm 1,2$ vs. $\mathrm{Pl}=1,9 \pm 2,0, \mathrm{P}<0,05)$. As características de personalidade e ansiedade foram semelhantes nos dois grupos, embora a ansiedade de estado tenha tendido a ser maior no grupo PR $(52 \pm 5$ vs. $49 \pm 4, p=0,06)$. Em conclusão, a prática regular do condicionamento físico parece reduzir os sintomas de stress e tende a melhorar a qualidade de vida profissional. Por outro lado, essa prática não modifica as características de personalidade dos praticantes.

\section{COMPARAÇÃO DE TESTES ERGOESPIROMÉTRICOS REALIZADOS EM CICLOSSIMULADOR E EM BICICLETA ERGOMÉTRICA}

Edenilson S. Carvalho Jr., Turíbio L. Barros Neto, Marcelo C. César, Alexandre L. G. Santos, André P. Scheneider, Liane Beretta.

Centro de Medicina da Atividade Física e do Esporte-CEMAFE, Escola Paulista de Medicina - Universidade Federal de São Paulo.

Um treinamento visa a adaptar um organismo para melhor realizar determinada tarefa. A especificidade, um dos princípios do treinamento, consiste em utilizar grupos musculares específicos para determinada modalidade. Para verificar o quanto a especifidade altera o resultado de um teste ergoespirométrico foram comparados, em indivíduos treinados, os principais índices de avaliação cardiorrespiratório por testes ergoespirométricos em bicicleta ergométrica e em ciclossimulador (ergômetro mais específico, por ser mais próximo a atividade praticada pelos atletas). Para testar os índices de avaliação física cardiorrespiratória, nove triatletas amadores treinados ( 6 do sexo masculino e 3 do sexo feminino), com idades variando entre 18 e 33 anos de idade, peso $70 \mathrm{Kg} \pm 9,69$ e altura de $1,75 \mathrm{~cm} \pm 7,70$, foram submetidos a dois testes de esforço em cicloergômetro com medida direta do consumo de oxigênio, protocolo com aquecimento de $3 \mathrm{~min}$. seguido por estágios de $1 \mathrm{~min}$. de duração até a exaustão. Na bicicleta ergométrica (CYBEX), a carga inicial foi de 100 watts (sexo masc.) e 50watts (sexo fem.) com incrementos de 50 watts a cada estágio; no ciclossimulador (CATEYE), a carga inicial foi de $1 \%$ (masc.) e $0 \%$ de inclinação (fem.) com incrementos de $1 \%$ cada estágio. Foram determinados Consumo Máximo de Oxigênio (VO2 max) e Limiar Anaeróbio Ventilatório (LA) por analisadores de gases (AMATEK-USA) e sistema metabólico VACUMED VISTA TURBOFIT-USA, freqüência cardíaca máxima (FC max) e do limiar anaeróbio ( $F C L A)$ por sistema de telemetria POLAR-USA.

Resultados: $\quad \mathrm{OO} \max$

Ergométrica

Ciclossimulador $\quad 62,88 \pm 5,13$

$\mathrm{p} /$ (teste t)

Ergométrica

Ciclossimulador

$\mathrm{p} /$ (teste t)

Os resultados indicam que a bicicleta ergométrica pode ser utilizada para avaliação física cardiorrespiratória de triatletas com a mesma precisão do ciclossimulador. 


\begin{tabular}{|c|c|c|c|}
\hline \multicolumn{4}{|c|}{$\begin{array}{l}\text { TESTE ERGOMÉTRICO NA ESTRATIFICAÇÃO DE R } \\
\text { APÓS INFARTO AGUDO DO MIOCÁRDIO NA ERA } \\
\text { TROMBOLÍTICA: INFLUÊNCIA DOS CRITÉRIOS DE } \\
\text { ANÁLISE SOBRE A ACURÁRIA DO TESTE. }\end{array}$} \\
\hline \multicolumn{4}{|c|}{$\begin{array}{l}\text { Renato B. O. Castro, Marcus V. Simões, João H. R. Ferreira, Paulo S. O. } \\
\text { Cavalcanti, Hilda W. Shida, Benedito C. Maciel, José A. Marin-Neto }\end{array}$} \\
\hline \multicolumn{4}{|c|}{ Divisão de Cardiologia, HC - FM - USP, Ribeirão Preto, SP, Brasil. } \\
\hline \multicolumn{4}{|c|}{$\begin{array}{l}\text { Objetivo: Analisar o impacto dos diferentes critérios de positividade utilizados na } \\
\text { análise do teste ergométrico convencional (TE) aplicado na estratificação de risco } \\
\text { (ER) após IAM sobre sua acurácia preditiva de eventos isquêmicos no seguimento a } \\
\text { longo prazo. } \\
\text { Métodos: Analisados } 142 \text { ptes consecutivos com IAM não complicado ( } 55 \pm 11 \text { anos, } \\
80 \% \text { masc., } 68 \% \text { tratados com trombólise endovenosa). Submetidos durante a } \\
\text { internação hospitalar a TE limitado por frequência cardíaca ( } 10 \pm 3 \text { dias) cujos critérios } \\
\text { de positividade foram: } 1 \text {. infradesnível do segmento } \mathrm{ST} \geq 1 \mathrm{~mm} \text {, horizontal ou } \\
\text { descendente; } 2 \text { angina; } 3 \text {. queda da pressão arterial no esforço; } 4 \text {. tolerância de } \\
\text { carga < } 6 \mathrm{METS} \text {; } 5 \text {. arritmia ventricular complexa. O resultado global do teste e a } \\
\text { presença dos diferentes critérios de positividade foram correlacionados com a } \\
\text { ocorrência de eventos isquêmicos no prazo médio de seguimento de } 25 \text { meses: angina } \\
\text { instável (20\%), reinfarto ( } 6 \% \text { ), morte cardíaca }(6 \%) \text {. Utilizou-se o teste de Qui-quadrado } \\
\text { para análise estatística com nível de significância p }<0,05 \text {. } \\
\text { Resultados: O TE foi positivo, pela evidência de qualquer critério, em } 49 \% \text { dos } \\
\text { ptes. A tabela abaixo apresenta a correlação com a ocorrência de eventos conforme } \\
\text { os critérios de positividade observados: }\end{array}$} \\
\hline Variável & Com eventos $(n=35)$ & Sem eventos $(n=107)$ & $\mathbf{P}$ \\
\hline $\begin{array}{l}\mathrm{ST} \geq 1 \mathrm{~mm} \\
\text { Carga }<6 \text { METS } \\
\text { Angina } \\
\text { Queda da PA } \\
\text { Arritmia ventricular } \\
\text { Global }\end{array}$ & $\begin{array}{l}13(37 \%) \\
5(14 \%) \\
4(11 \%) \\
8(23 \%) \\
2(6 \%) \\
18(51 \%)\end{array}$ & $\begin{array}{l}28(27 \%) \\
23(21 \%) \\
7(6 \%) \\
6(6 \%) \\
2(2 \%) \\
51(49 \%)\end{array}$ & $\begin{array}{l}0,24 \\
0,35 \\
0,35 \\
0,003 \\
0,23 \\
0,70\end{array}$ \\
\hline \multicolumn{4}{|c|}{$\begin{array}{l}\text { O comportamento anormal da pressão arterial mostrou correlação com ocorrência } \\
\text { de eventos: } \mathrm{S}=23 \% \text {; } E=94 \% ; \mathrm{VP}+=57 \% \text {; } \mathrm{VP}-=79 \% \text {. } \\
\text { Conclusões: Critérios individuais de análise do TE na avaliação de risco pós IAM } \\
\text { podem apresentar diferenças significativas na acurácia preditiva de eventos. O } \\
\text { parâmetro vinculado à disfunção ventricular apresentou rendimento superior aos } \\
\text { demais na casuística estudada. }\end{array}$} \\
\hline
\end{tabular}

\section{ANÁLISE COMPARATIVA ENTRE TESTE ERGOMÉTRICO PRECOCE E A CINECORONARIOGRAFIA NA ESTRATIFICAÇÃO DE RISCO APÓS INFARTO AGUDO DO MIOCÁRDIO NÃO COMPLICADO NA ERA TROMBOLÍTICA.}

Marcus V. Simões, Renato B. Pereira de Castro, Elias Melo Ayres-Neto, José L. AttabSantos, Benedito C. Maciel, Rogério T. Tumelero, André Schmidt, José A. Marin-Neto.

Divisão de Cardiologia, HC-FMRP-USP.

Objetivo: Parece reduzida a capacidade de detecção eletrocardiaográfica de isquemia miocárdica nos pacientes pós-IAM com uso de trombólise química (TQ). A ocorrência de acometimento coronariano multivascular também é menor do que o observado na era prétrombólise. Intenta-se aquilatar o poder prognóstico do teste ergométrico submáximo precoce (TESP) e da cinecoronariografia (CC) neste contexto.

Casuística e métodos: 142 ptes consecutivos com IAM não complicado (55+11 anos), endo $68 \%$ destes recebido TQ No prazo médio de 10 dias (extremos de 5 a 17), foram submetido aTESP, sendo critérios de positividade: infradosnivl do segmento ST hoizonta submetidos a TESP, sendo crierios de positividade: infradesnivel do segmento ST horizontal ( $>1 \mathrm{~mm}$ ou descendent, desencadea de esto isquêmicos ocorridos em período médio de 25 meses de seguimento: angina instáve $(20 \%)$, reinfarto $(6 \%)$, morte de causa cardíaca $(6 \%)$.

Resultados: Curvas de sobrevida livre de eventos de Kaplan-Meyer, conforme resultado da CC e TESP.
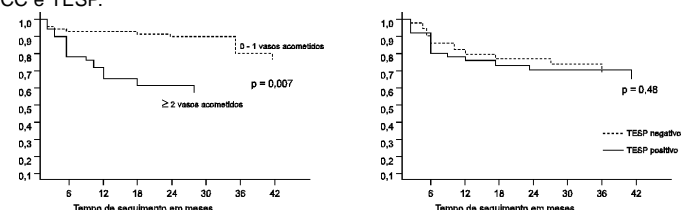

O TESP foi positivo em 69 ptes $(49 \%)$, tendo obtido um tempo de sobrevida livre de eventos (TSLE) de $43 \pm 3 \mathrm{~m}$ e $46 \pm 3 \mathrm{~m}$ para o TESP positivo e negativo, respectivamente eventos (TSLE) de $43 \pm 3 \mathrm{~m}$ e $46 \pm 3 \mathrm{~m}$ para o TESP positivo e negativo, respectivamente
$(\mathrm{p}=0,48)$. A detecção de acometimento de $\geq 2$ vasos à CC ocorreu em 49 ptes $(34 \%)$, tendo-se correlacionou-se com TSLE $=37 \pm 4 \mathrm{~m}$, estatisticamente menor que os pacientes tendo-se correlacionou-se com TSLE $=37 \pm 4 \mathrm{~m}$, estatisticamente menor que os pacientes
exibindo acometimento de $0-1$ vaso (93 ptes, $66 \%)$, que foi de $48 \pm 2 \mathrm{~m}-(\mathrm{p}=0,007)$. O acometimento multiarterial à $\mathrm{CC}$ identificou corretamente $18(51 \%)$ dos ptes que vieram a apresentar eventos (VP+=37\%; VP-=82\%).

Conclusão: O TESP usado para estratificação prognóstica contribui pouco, comparativamente à CC, para predição de eventos isquêmicos em população pós-IAM em que se une rotineiramente $\mathrm{TQ}$.

\section{O TESTE ERGOMÉTRICO CONVENCIONAL É CAPAZ DE IDENTIFICAR PORTADORES DE CORONARIOPATIA MULTIARTERIAL EM SOBREVIVENTES AO INFARTO AGUDO DO MIOCÁRDIO NA ERA TROMBOLÍTICA?}

Renato B. P. Castro, Marcus V. Simões, Rogério T. Tumelero, Elias M. Ayres-Neto, José L. Attab-Santos, André-Santos, André Schmidt, Benedito C. Maciel, José A. Marin-Neto

Divisão de Cardiologia, HC - FM - USP, Ribeirão Preto, SP, Brasil.

Objetivo: Investigar a acurácia do teste ergométrico convencional (TE) aplicado na estratificação de risco após IAM em detectar pacientes portadores de doença arterial coronária de múltiplos vasos.

Métodos: Analisados 142 ptes consecutivos com IAM não complicado ( $55 \pm 11$ anos, $80 \%$ masc., $68 \%$ tratados com trombólise endovenosa). Submetidos durante a internação hospitalar a TE limitado por frequência cardíaca ( $10 \pm 3$ dias) cujo critério de positividade foi a ocorrência de pelo menos um dos seguintes: 1 infradesnivel do segmento ST $\geq 1 \mathrm{~mm}$, horizontal ou descendente; 2 angina; 3 . queda da pressão arterial no esforço; 4 . tolerância de carga < 6 METS; 5 . arritmia ventricular complexa. No estudo cinecoronariográfico realizado antes da alta hospitalar ou no intervalo de 30 dias após, foram consideradas significativas as lesões cousando redução do diâmetro luminal $\geq 50 \%$. Para análise estatística o resultado do TE foi correlacionado com a extensão do acometimento arterial coronário mediante uso do teste de Quiquadrado.

Resultados: O TE foi positivo em $49 \%$ dos ptes. Doença coronária uniarterial foi identificada em 57 ptes $(41 \%)$, biarterial em $33(23 \%)$, multiarterial $16(11 \%)$. A correlação entre os resultados é resumida abaixo:

\begin{tabular}{|c|c|c|c|}
\hline \multirow[t]{2}{*}{ Resultado do TE } & \multicolumn{2}{|c|}{ Extensão da coronariopatia } & \multirow[b]{2}{*}{$\mathbf{P}$} \\
\hline & $\geq 3$ vasos & $<3$ vasos & \\
\hline Positivo & 11 & 58 & 0,07 \\
\hline Negativo & 5 & 68 & \\
\hline
\end{tabular}

Comentários e conclusões: $O$ teste egométrico apresentou forte tendência de correlação com a extensão do acometimento arterial coronário, exibindo uma satisfatória sensibilidade e alto valor preditivo negativo na detecção de coronariopatia multiarterial.

\section{AVALIAÇÃO DE MÉTODOS DE PREDIÇÃO DA CAPACIDADE AERÓBIA MÁXIMA OBTIDA ATRAVÉS DE} TESTE ERGOMÉTRICO EM ESTEIRA ROLANTE

Barbara M. Durão, Graciema S. Porphírio, Oswaldo C. A. Andrade, André S. F. Serra, Luciano Loos, Salvador M. Serra. Instituto Estadual de Cardiologia Aloysio de Castro / R.J.

Objetivo: Identificar o método mais adequado na estimativa da capacidade aeróbia máxima (VO2 máx), possibilitando a realização de teste ergométrico máximo (TE. máx) no tempo idealmente preconizado de 8 a 12 minutos.

Material e método: 18 indivíduos saudáveis (44\% masc.; 39 anos +/- 8) foram submetidos a 2 TE máx em esteira rolante utilizando-se protocolos de Bruce e Rampa, estimando-se o VO2 alcaçado através das equações do "American College of Sports Medicine". Previamente foram aplicados 2 questionários (VSAQ e Duke) e 2 equações (Jones e Froelicher) objetivando a predição daqueles valores.

Resultados: Em ml O2 / Kg / min; Bruce: 41,7+/-5,5 e Rampa: 42,9+/-5,7 (NS). Comparados aos valores preditos: VSAQ: 42,7 $+/-4,6$ (NS); Duke: $33,4+/-1,9(p<0,0001)$; Jones: $35,7+/-4,6$ $(p<0,002)$; Froelicher: $34,3+/-5,1(p<0,0001)$.

Conclusão: A aplicação do questionário VSAQ mostrou ser o único método eficiente em predizer a capacidade aeróbia máxima avaliada através de teste ergométrico em esteira rolante. 


\section{RAMPA SOB CONTROLE MANUAL: ALTERNATIVA DE PROTOCOLO INDIVIDUALIZADO PARA TESTE ERGOMÉTRICO}

Barbara M. Durão, Graciema S. Porphírio, Oswaldo C. A. Andrade, Andre S.F. Serra, Luciano Loos, Salvador M. Serra

Instituto Estadual de Cardiologia Aloysio de Castro / R.J.

Objetivo: Possibilitar a aplicação de protocolo de rampa por laboratórios de ergometria que não disponham de equipamentos computadorizados específicos. Material e métodos: 18 indivíduos saudáveis (44\% masc., 39 anos +/- 8) foram submetidos a 2 testes ergométricos (TE) utilizando alternadamente protocolo de Bruce e Rampa sob controle manual denominado "de Castro", sendo este selecionado entre 6 opções baseadas no VO2 máximo estimado através de aplicação de questionário. O VO2, para ambos os protocolos, foi calculado pelas equações do "American College of Sports Medicine". O tempo alvo de exercício foi de 8 a 12 minutos. Todos os TE foram aplicados pelos mesmos profissionais e equipamentos no mesmo horário da manhã e no período de 7 dias.

Resultados: $100 \%$ dos indivíduos concluíram ambos os protocolos a nível máximo. Foi observado para o protocolo de Bruce e Rampa manual "de Castro", respectivamente: tolerância em METS: $11,9+/-1,6$ e 12+/-1,6 (NS); tempo em minutos: $11,4+/-1,8$ e $12+/-1,8$ ( $p=0,002)$; FC (bpm) $181+/-14$ e 180+/-15 (NS); duplo produto máximo: $31161+/-6746$ e $28185+/-6236(\mathrm{p}=0,002)$; escala de Borg (aos 7 METS): $2,8+/-1,9$ e 3,6+/-1,8 $(\mathrm{p}=0,009)$. Ao término do TE 16 indivíduos (89\%) referiram melhor adaptação ao protocolo de Rampa manual "de Castro".

Conclusões: 1 . O protocolo de Rampa é passível de aplicação sob controle manual; 2. Os valores de VO2 máximo estimados por questionário foram equivalentes aos obtidos no teste ergométrico; 3 . A tolerância e a frequência cardíaca máximas foram semelhantes em ambos os protocolos; 4 . O protocolo de Bruce determinou maior duplo produto às custas de níveis mais elevados de pressão arterial sistólica; 5 . Embora a sensação de cansaço a nível submáximo tenha sido menor durante o protocolo de Bruce, houve melhor adaptação com o protocolo de Rampa manual "de Castro".

\section{TESTE ERGOMÉTRICO SINTOMA LIMITANTE PRECOCE PÓS-INFARTO: VALOR DO TEMPO DE ENDURANCE.}

Wagner Leite, Luciano Vacanti, Antonio C. Carvalho, José E. Grecco, Orlando Campos Fo, Dirceu Santos Fo, Japy Oliveira Fo․

UNIFESP - Escola Paulista de Medicina - SP

Objetivo: Verificar o valor de tempo de endurance (T. End.) no teste ergométrico (TE) como indicativo do risco potencial, em série de paciente (pt) consecutivos pós-infarto do miocárdio (IAM), de boa evolução clínica, $\mathrm{FE}>0,50$ (ecocardiograma), na fase pré-alta hospitalar.

Material e Métodos: 60 pt pós-IAM realizaram TE, cinecoronariografia (cine) e holter $24 \mathrm{hs}(\mathrm{H}-24)$. Os pt eram 46 homens, $50 \pm 20$ anos, sendo 35 trombolisados (SK) e 25 IAM anterior sem contra indicações para TE.

TE foi sintoma limitante, 12 derivações em protocolo de Naughton.

Critérios: TE (consenso S.B.C.); lesões obstrutivas críticas (LOC) (> $70 \%$ luz arterial), T. End. baixo ( $<15$ min. $<6$ mets), significância estatística $(\mathrm{P}<0,05)$

Resultados: TE realizaram-se sem acidentes, sendo $45 \%$ anormais e $20 \%$ isquêmicos. $\mathrm{No} \mathrm{H}-24$, detectaram-se arritmias ventriculares em $83 \%$ (30\% arritmias complexas). $\mathrm{Na}$ cine, houve, $35 \%$ de pt com LOC multiarteriais e $40 \%$ de pt com LOC uni-arterial. Nos TE de baixo T End. $(n=23)$, ocorreram 8 TE isquêmicos (35\%), 20 TE anormais (87\%), 12 pt com LOC multiarteriais (52\%); nos TE com T End. normal ocorreram 6 TE isquêmicos (16\%), 10 TE anormais (27\%) e 9 LOC multiarteriais (24\%), sendo estas diferenças significativas. Não houve diferença significativa quanto a presença de arritmias complexas em TE com T End baixo ou normal.

Conclusão: Os autores destacam o valor do tempo de endurance como variável indicativa de potencial de risco em pt de boa evolução pós-IAM pré alta hospitalar.

\section{RESPOSTAS CARDIORESPIRATÓRIAS APÓS TRANSPLANTE CARDÍACO EM EXERCÍCIOS LEVES A MODERADOS.}

Ana Salles, Japy Oliveira Fo, Turibio Barros, Dirceu Almeida, Antonio Carlos Carvalho, Yara Juliano, Enio Buffolo, Dirceu Santos Fo

UNIFESP - Escola Paulista de Medicina - SP

Objetivo: Avaliar as respostas de pacientes (pt) com transplante cardíaco (TXC) durante exercícios leves a moderados (até $5 \mathrm{MET}, 17,5 \mathrm{ml} \mathrm{O} / \mathrm{Kg}$ $\min )$.

Métodos: Submeteram-se a testes ergoespirométricos (TEE) 9 pt com TXC (GI) pareados por sexo, idade, peso e altura a 9 indivíduos sedentários aprarentemente sadios (GII). Os pt eram homens, com $48 \pm 12$ anos, em TFI (NYHA), após $23 \pm 21$ meses de TXC. TEE foram realizados em cicloergômetro com incrementos de $5 \mathrm{~W} / \mathrm{min}$ até a exaustão, após estágio incial de 3 min a $25 \mathrm{~W}$. Consumo de $\mathrm{O}_{2}$ foi feito em sistema metabólico VACUMED e análise estatística pelo teste de Mann - Whitney $(P \leq 0,05)$.

Resultados: Em potência de $40 \mathrm{~W}$, (abaixo do LA) correspondente a exercícios leves a moderados, as variáveis cardiorrespiratórias apresentam, respectivamente, nos grupos I e II os seguintes valores: $\mathrm{VO}_{2}\left(\mathrm{ml} \mathrm{O}_{2} \mathrm{Kg} /\right.$ $\mathrm{min}$ ), 12,34 x 12,38 (NS); VE BTPS, (1/min) 29,28 × 25,40 (NS); VE O $32,60 \times 28,85$ (NS); VE CO $, 37,58 \times 38,84$ (NS); FC (bpm), $119 \times 114$ (NS); tempo de endurance (min) $3 \times 3$ (NS); Potência (W) $40 \times 40$ (NS).

No limiar anaeróbio, foram significativos apenas as diferenças relativas, ao $\mathrm{VO}_{2}$, Potência e tempo de endurance, respectivamente nos grupos I e II; $14,22 \times 19,51{ }^{*} \mathrm{ml} \mathrm{O} 2 \mathrm{Kg} / \mathrm{min} ; 5 \times 9{ }^{*} \min ; 51 \times 76$ * W.

Conclusões: Os autores concluíram que em potência de $40 \mathrm{~W}$ o desempenho dos pt TXC foi semelhante aos pares normais, evidenciando os benefícios do TXC durante atividades físicas habituais.

\section{FREQÜÊNCIA CARDÍACA NO LIMIAR ANAERÓBIO EM INSUFICIÊNCIA CARDÍACA}

Japy Oliveira Fo, Xiomara Salvetti, Dirceu Almeida, Ana Salles, Fernando Torres, Turibio Barros, Antonio Silva, Dirceu Santos Fo.

\section{UNIFESP - Escola Paulista de Medicina - São Paulo}

Objetivo: Avaliação da freqüência cardíaca $(F C)$ no limiar anaeróbio (LA) em pacientes (pt) com insuficiência cardíaca compensada (ICCo)

Casuística: 18 pt, $46 \pm 8$ anos, 13 homens, com doença coronária (10 pt), cardiomiopatia dilatada (3 pt) e doença de Chagas (5 pt), em tipo funcional (NYHA) 1 (2 pt), III (3 pt), IV (7 pt), em uso de digital, diuréticos e inibidores da (ECA).

Métodos: Teste ergoespirométrico (TEE) em protocolo de rampa, em cicloergômetro (aquecimento $25 \mathrm{~W}, 3 \mathrm{~min}$; acréscimo $5 \mathrm{~W} /$ $\mathrm{min}$ ) dob monitorização (CM5), com medida das variáveis em circuito aberto, em sistema metabólico Vista CX - VACUMED, com medidas cada 15 segundos (Programa Turbofit); eletrocardiograma convencional (ECG) em repouso.

Resultados: TEE ocorreram sem intercorrências interrompidos por exaustão em todos os casos. Variáveis aferidas: $\mathrm{VO}_{2}$ pico 17 $\pm 4 \mathrm{m1O}_{2} / \mathrm{Kg} / \mathrm{min} ; \mathrm{LA} 11 \pm 2,5 ; \mathrm{FC}$ pico $141 \pm 24 ; \mathrm{FC}$ LA $122 \pm$ 16; FC controle (TEE) $87 \pm 21$; FC (ECG) $91 \pm 4$; LA / VO 2 pico 67 \pm 11; FC LA/FC $0,87 \pm 0,07$. Os coeficientes para a fórmula de Karvonem para treinamento físico no LA foram de 0,88 $\pm 0,07$ (FC ECG), $0,80 \pm 0,18$ (FC controle TEE).

Conclusão: Os autores relatam valores elevados para FC LA em relação ao $\mathrm{FC}$ pico em pt portadores de ICCo com níveis variáveis entre 75 a $98 \%$. 


\section{CORRELAÇÃO CLINICA-ELETROCARDIOGRÁFICA- RADIOLÓGICA E ERGOMÉTRICA DE HIPERTENSOS PORTADORES DE PRECORDIALGIA EM RIB. PRETO - SP}

Cesarino, E.J.; Roselino, F.J.V.; Viana, L.A.L.; Lanchoti, V.L. Associação Ribeirãopretana de Ensino, Pesquisa e Assistência ao Hipertenso; SMS-Rib. Preto e FCFRP-USP.

O objetivo do trabalho foi correlacionar as características clinicas com os achados do eletrocardiograma, RX de Tórax PA e teste ergométrico de hipertensos com história de precordialgia.

No período de fev. 90 a fev. 92 foram acompanhados 564 indivíduos hipertensos, sendo 312 mulheres (55,31\%); 77 (13,65\%) referiam precordialgia na $1^{\mathrm{a}}$ consulta, sendo $62(80,51 \%)$ mulheres, a idade variou de 28 a 84 anos (média 53,2 anos); 52 indivíduos $(67,53 \%)$ tinham antec. familiar de infarto do miocárdio; 19 $(24,67 \%)$ diabéticos e $24(31,16 \%)$ fumantes. O ECG revelou alt. repolarização ventricular em 36 indivíduos (46,75\%); área inativa em $10(12,98 \%)$ e sobrecarga de câmara em $16(20,77 \%)$. O RX revelou cardiomegalia em 28 indivíduos $(36,36 \%)$. O teste ergométrico revelou desnível do segmento ST compatível com resposta isquêmica em $10(12,98 \%)$ e dor durante o esforço em 6 $(7,79 \%)$, presença de resposta hiperreatora da pressão arterial sistólica e/ou diastólica em 35 indivíduos (45,45\%).

Conclui-se que houve predomínio de mulheres hipertensas referindo percordialgia com diversas alterações eletrocardiográficas e radiológicas, com baixa ocorrência de sinais eletrocardiográficos sugestivos de resposta isquêmica do miocárdio na prova de esforço. Apesar da importante frequência de fatores de risco coronários nestes pacientes, o teste ergométrico conseguiu reproduzir a dor precordial em pequeno $\mathrm{n}^{\circ}$ de indivíduos.

\section{TESTE DINÂMICO VERSUS TESTE ESTÁTICO: COMPARAÇÃO DAS VARIÁVEIS HEMODINÂMICAS E METABÓLICAS.}

José A. Caldas Teixeira, Washington B. Araújo, João Alexandre R. Assad, Suely C. Soares

\section{UFF/FITCENTER/CARDIOLAB}

O objetivo do estudo foi comparar as variáveis hemodinâmicas máximas e algumas metabólicas entre um teste ergométrico (TE) dinâmico máximo, com um exercício estático (EE) máximo utilizando grupamentos musculares equivalentes.

Material e métodos: Foi utilizado um TE sintoma limitante em esteira pelo protocolo de Bruce e um isoergômetro de membros inferiores para uma extensão estática sustentada de ambas as pernas (EESAP). A EESAP foi realizada a $50 \%$ da FMV (força máxima voluntária) em posição supina durante 1 minuto ou até a fadiga. As variáveis analisadas foram frequência cardíaca (FC), TA Sistólica (PAS)., PA Diastólica (PAD)., duplo produto (DP), consumo miocárdio de Oxigênio (MVO2), Delta PAS. Delta PAD. A amostra constou de 18 indivíduos com idade média 50,9 anos (+- 10,8), sexo masc. Massa corporal de $70 \mathrm{Kg}(+-10,3)$, estatura $1,69 \mathrm{~m}(+-0,9)$. VO2 Mx. em esteira 43,9 (+- 5,9), FMV na EESAP de $58,7 \mathrm{Kg}$, com $50 \% 29,3 \mathrm{Kg}(\times 2,5 \mathrm{Kg})$.

Resultados: $p<0,05$, valores médios e desvio padrão.

\begin{tabular}{llllllll}
\hline & FCMx & PAS Mx & PAD Mx & DP Mx & D PAS & D PAD & MVO2 \\
\hline Esteira & 174,2 & 198,6 & 85,9 & 34670 & 74,8 & 0,3 & 42,2 \\
& $+-18,1$ & $+-19,9$ & $+-11,4$ & +-5416 & $+-16,7$ & $+-14,4$ & $+-7,6$ \\
EESAP & 156,6 & 221,9 & 143,4 & 33855 & 91,4 & 31,4 & 41,1 \\
& $+-19,7$ & $+-35,1$ & $+-15,2$ & +-6558 & $+-37,3$ & $+-17,6$ & $+-9,2$ \\
p & 0,002 & 0,03 & 0,00006 & s/sig. & s/sig. & 0,00006 & s/sig. \\
\hline
\end{tabular}

Conclusões: 1) Maior resposta pressórica no Exercício Estático (EE), tanto da PA Sist. (11,6\%), quanto da PA Diast. que foi $59,2 \%$ maior. 2) Maior resposta cronotrópica no TE, com o EE atingido somente $85,8 \%$ em relação ao dinâmico. 3) Os parâmetros metabólicos que retratam o estresse miocárdico e seu consumo de $\mathrm{O} 2$ não mostram diferença significativa.

\section{ANÁLISE DO COMPORTAMENTO DA FREQUÊNCIA CARDÍACA NA FASE DE RECUPERAÇÃO DE PROVAS CICLOERGOMÉTRICAS COMO PROVÁVEL INDICATIVO DE CAPACIDADE FÍSICA FUNCIONAL}

Cesarino, E.J.; Vichi, F.L.; Franco, M.A.P.; Lanchoti V.L.

Departamentos de Clinica Médica e Matemática aplicada a Biologia da FMRP-USP e FCFRP-USP.

O objetivo deste estudo consistia na observação do comportamento da frequência cardíaca (FC) durante a fase de recuperação de testes cicloergométricos convencionais em indivíduos sedentários e atletas.

O material e método utilizado foram 20 indivíduos sedentários (Grupo I) e 20 atletas (Grupo II), sendo estes últimos constituídos por 7 futebolistas profissionais e 13 basquetebolistas, do sexo masculino, não fumantes, não obesos, sem evidência de cardiopatias, assintomaticos, sem uso de drogas de ação cardiovascular, voluntários, com idade de 18 a 31 anos (média: 23, 35 anos, DP: 3,8). O teste cicloergométrico foi contínuo com cargas progressivas, submáximo, para que todos interrompessem o teste numa FC semelhante, usando a derivação MC5 e o protocolo de Astrand-Ryhming. A FC foi obtida a partir da média das medidas de 3 intervalos RR antes, durante e após o esforço nos tempos 30 seg., 1 min., 2 min., 4 min., 6 min., 8 min. e 10 min. A FC de repouso foi inferior no grupo II $(p=0,05)$; a duração do esforço maior no grupo II $(p<0,001)$; consumo indireto de oxigênio maior no grupo II $(\mathrm{P}=0,002)$; a $\mathrm{FC}$ teve menor incremento durante o esforço e decréscimo mais rápido na fase de recuperação no grupo II.

Conclui-se que o comportamento da FC na fase de recuperação de provas cicloergométricas convencionais pode constituir num indicativo adicional de capacidade física funcional.

\section{PAPEL DO TESTE ERGOMÉTRICO PRECOCE NA AVALIAÇÃO DE PACIENTES COM DOR TORÁCICA NA SALA DE EMERGÊNCIA}

Renato Macaciel, Roberto Bassan, Ricardo Vivácqua, Salvador Serra, Marcelo Miranda, Augusta Leite, em nome dos investigadores do Projeto Dor Torácica

Fundamento: Muitos pacientes (pts) antendidos com Dor Torácica (DT) na Sala de Emergência (SE), recebem alta sem um diagnóstico, e sem uma estratificação de risco.

Objetivos: 1- Estabelecer a acurácia diagnóstica do Teste Ergométrico (TE) precoce para avaliação de pts admitidos na SE com DT e suspeita de Insuficiência Coronariana Aguda (ICA).

Métodos: Estudo prospectivo com 136 pts consecutivos, idade = $59,4 \pm 15,7 ; \mathrm{H}=64.7 \% \Delta t$ médio $=3,8 \mathrm{~h}$ (início da DT - atendimento), com DT nas últimas $12 \mathrm{~h}$. Atendidas na SE de um hospital emergencial, período nov 96/jan 97. Foram excluídos pts com diagnóstico de IAM, 26 pts (19.11\%) e 110 pts elegíveis para o TE; 30 (22\%) pts tiveram o diagnóstico final de Angina Instável (AI) e 80 pts (58\%) de ausência de ICA. O TE foi sintoma limitante, com protocolo de incrementados baixa carga.

Resultados: Dos 110 pts elegíveis para o TE 19 pts (17.2\%) realizaram o TE. Destes 05 pts tiveram o diagnóstico clínico pré-teste de Al e 4 tiveram TE positivo com tempo de chegada no hospital - realização do TE $=24.2 \pm 14.7 \mathrm{~h}$. Dos 14 pts com diagnóstico clínico de ausência de ICA, nenhum teve TE positivo; tempo de chegada ao hospital e realização do TE $(21,9 \pm 19,4$ h). Não ocorreram complicações com TE.

Conclusão: 1- O TE mostrou-se um método seguro na avaliação de pts com DT na Sala de Emergência. 2-O TE mostrou elevada sensibilidade $(80 \%)$ e excelente especificidade $(100 \%)$, valor de previsão positivo $(100 \%)$ e valor de precisão negativa (93\%) para o diagnóstico de Al e de ausência de ICA. 


\section{CARACTERÍSTICAS DO TESTE ERGOMÉTRICO} PRECOCE EM PACIENTES ATENDIDOS NA SALA DE EMERGÊNCIA COM DOR TORÁCICA: ANGINA INSTÁVEL VS AUSÊNCIA DE INSUFICIÊNCIA CORONARIANA AGUDA.

Renato Macaciel, Roberto Bassan, Salvador Serra, Augusta Campos, Marcelo Miranda, Ricardo Vivácqua, em nome dos Investigadores do Projeto Dor Torácica

Hospital Pró-Cardíaco - Rio de Janeiro - RJ.

Fundamentos: Pacientes (pac) que são diagnosticados como portadores de angina instável (Al), podem estabilizar rapidamente, ainda assim, torna-se imperativo determinar a presença de isquemia residual.

Objetivos: Avaliar a resposta ao esforço de pac atendidos com Dor Torácica (DT) e diagnóstico clínico pré-teste de Al vs ausência de insuficiência coronariana aguda (ICA).

Métodos: Estudo prospectivo com 136 pac consecutivos idade $=59,4 \pm 15,7$, homens $=64,7 \% ; \Delta t$ médio de $3,8 \mathrm{~h}$ (inicio da DT - atendimento) com DT nas últimas $12 \mathrm{~h}$ na SE deste hospital emergencial no período de $11 / 96$ a 01/97. Foram excluídos 26 pac com diagnóstico de IAM, restando 110 elegíveis para o TE. Destes, $30(27 \%)$ tiveram diagnóstico clínico final pré-teste de Al e 80 (73\%) de ausência de (ICA).

Resultados: Dos 110 pacientes pac elegíveis para o TE, $19(17,2 \%)$ realizaram o teste. Destes, 5 tinham o diagnóstico clínico pré-teste de Al e 4 tiveram TE isquêmico, com tempo de chegada ao hospital e realização do $T E=24,2 \pm 14,7 \mathrm{~h}$. Três tiveram resposta hipertensiva. Dos 14 pac. com diagnóstico clínico pre-teste de ausência de ICA, nenhum teve TE positivo, com tempo de chegada ao hospital realização do $\mathrm{TE}=21,9 \pm 19,4$. Parâmetros avaliados: idade: $\mathrm{Al}=57,0 \pm 2,8$; ausência de $\mathrm{ICA}=47,1 \pm 11,5$. Interrupção do TE: $\mathrm{Al}=2 \mathrm{c} /$ angor, $3 \mathrm{c} /$ cansaço; ausência de ICA= $13 \mathrm{c} /$ cansaço, $1 \mathrm{c} /$ dor MMII; tolerância: $\mathrm{Al}=7,4 \pm 3,9 \mathrm{METS}$; ausência de $\mathrm{ICA}=12,32$ $\pm 3,0(p=0,01)$. Duplo produto: $A \mathrm{~A}=34420 \pm 4624$, ausência de $\mathrm{ICA}=30287 \pm 6877$ (NS). ECG de esforço isquêmico: $\mathrm{Al}=60 \%$, sem $\mathrm{ICA}=0$.

Conclusões: 1- $\mathrm{Pac}$. com Al, assintomáticos há $24 \mathrm{~h}$ podem ser submetidos a TE precoce. 2- A maioria destes pac apresenta isquemia ao esforço e menor gasto calórico (METS) em relação aos pac sem ICA, mas alcançam adequado DP, principalmente, as custas da maior elevação pressórica; $3-0$ ECG de esforço mostrou excelente especificidade para de Insuficiência Coronariana Aguda.
PREVALÊNCIA DO DISTÚRBIO DO RITMO EM ATLETAS COMPETITIVOS PORTADORES DE PROLAPSO DE VÁLVULA MITRAL

Nabil Ghorayeb, Giuseppe S. Dioguardi, Patrícia Smtih, Cláudio A. Baptista, Carlos C. Luiz, Luis E. Mastrocolla, Stella Grespan, Felício Savioli Neto, Michel Batlouni.

Durante o período de janeiro a julho de 1997, 74 atletas competitivos, sendo 63 masculinos com idade entre 15 a 35 anos, foram examinados segundo o protocolo de avaliação cardiológica de atletas que consiste em exame clínico, laboratorial, eletrocardiograma (ECG) de repouso, teste ergométrico (TE), ecodopplercardiograma e eletrocardiograma (ECG) dinâmico de $24 \mathrm{~h}$ nos casos com indicação.

O prolapso de válvula mitral (PVM) foi diagnosticado pelo ECO em 16 atletas sendo 12 masculinos, todos sem degeneração mixematosa ou anormalidades associadas. Desses apenas 04 atletas tinham distúrbios de ritmo: ESSV no ECG pré-teste em 01 atletas e ESV monomórficas isoladas durante o TE em 03.

Nos atletas sem PM foram detectados distúrbio de ritmo, no TE 03 atletas foram detectados, 01 atleta com ESSV, 01 TPSV, 01 ESV e $01 \mathrm{com}$ ritmo juncional no ECG de repouso.

Em ambos os grupos os METS atingidos foram semelhantes (com PVM $=17 \pm 2.0$ e nos sem PVM $=18.5 \pm 2.4$ ).

Conclusão: Não houve diferenças estatísticas entre os dois grupos em relação às complexidades das arritmias. 